\title{
Molecular Beam Depletion: A New Approach
}

\author{
Manuel Dorado \\ CIRTA, Rotation and Torque Research Center, Theoretical Group, Madrid, Spain \\ Email: mdorado@cirta.es
}

Received 10 May 2014; revised 7 June 2014; accepted 1 July 2014

Copyright () 2014 by author and Scientific Research Publishing Inc.

This work is licensed under the Creative Commons Attribution International License (CC BY).

http://creativecommons.org/licenses/by/4.0/

(c) (i) Open Access

\begin{abstract}
During the last years some interesting experimental results have been reported for experiments in $\mathrm{N}_{2} \mathrm{O}$, NO, NO dimer, $\mathrm{H}_{2}$, Toluene and $\mathrm{BaFCH}_{3}$ cluster. The main result consists in the observation of molecular beam depletion when the molecules of a pulsed beam interact with a static electric or magnetic field and an oscillating field (RF). In these cases, and as a main difference, instead of using four fields as in the original technique developed by I.I. Rabi and others, only two fields, those which configure the resonant unit, are used. That is, without using the nonhomogeneous magnetic fields. The depletion explanation for I.I. Rabi and others is based in the interaction between the molecular electric or magnetic dipole moment and the non-homogeneous fields. But, obviously, the change in the molecules trajectories observed on these new experiments has to be explained without considering the force provided by the field gradient because it happens without using non-homogeneous fields. In this paper a theoretical way for the explanation of these new experimental results is presented. One important point emerges as a result of this development, namely, the existence of an, until now unknown, spin-dependent force which would be responsible of the aforementioned deviation of the molecules.
\end{abstract}

\section{Keywords}

Molecular Beam Magnetic Resonance, Molecular Beam Depletion, Larmor Precession, Helicity

\section{Introduction}

The molecular beam magnetic resonant (MBMR) technique has significantly contributed, as is well known, to the development of atomic and molecular physics (1). And it makes possible to measure de Larmor frequency of an atom or molecule in the presence of a magnetic field. In the original technique, developed by I.I. Rabi and others [1]-[3] the molecular beam is forced to pass through four different fields:

- A non-homogeneous polarizer field (A) where the molecules are prepared.

- A resonant unit (C) that consists of two, a static and an oscillating, fields.

- A non-homogeneous analyzer field (B). Only molecules in the prepared state reach the detector. 
- The two non-homogeneous magnetic fields A and B have opposite directions.

The molecular beam describes a sigmoidal trajectory and, finally, is collected in a detector (see Figure 1).

Rabi explained this effect in terms of spatial reorientation of the angular moment due to a change of state when the transition occurs.

In this case the depletion explanation is based in the interaction between the molecular magnetic dipole moment and the non-homogeneous fields.

$$
\boldsymbol{F}=\nabla(\boldsymbol{\mu} \cdot \boldsymbol{B})
$$

The force is provided by the field gradient interacting with the molecular dipolar moment (electric or magnetic). On the resonant unit the molecular dipole interact with both, homogeneous and oscillating, fields. When the oscillating field is tuned to a transition resonant frequency between two sub states, a fraction of the molecular beam molecules is removed from the initial prepared state. As a consequence, the dipolar moment changes as well as the interaction force with the nonhomogeneous analyzer field (B). As only molecules in the initial prepared state reach the detector the signal in the detector diminishes.

\section{New Experimental Results}

During the last years some interesting experimental results have been reported for $\mathrm{N}_{2} \mathrm{O}$, NO, NO dimer, $\mathrm{H}_{2}$ and $\mathrm{BaFCH}_{3}$ cluster [4]-[7]. The main result consists in the observation of molecular beam depletion when the molecules of a pulsed beam interact with a static electric or magnetic field and an oscillating field (RF) as in the Rabi's experiments. But, in these cases, instead of using four fields, only two fields those which configure the resonant unit (C), are used, that is, without using the non-homogeneous magnetic, A and B, fields. See Figure 2.

In a similar way, when the oscillating field is tuned to a transition resonant frequency between two sub states,

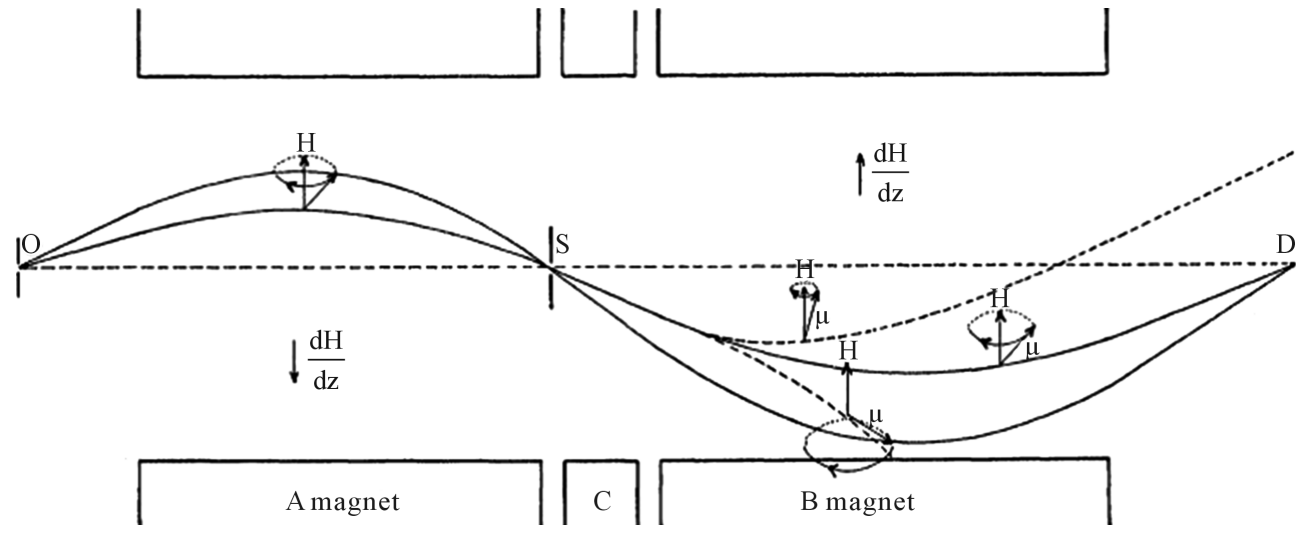

Figure 1. Typical path of molecules in a M.B.M.R experiment. The two solid curves show the paths of the molecules whose moments do not change when passing through the resonant cell.

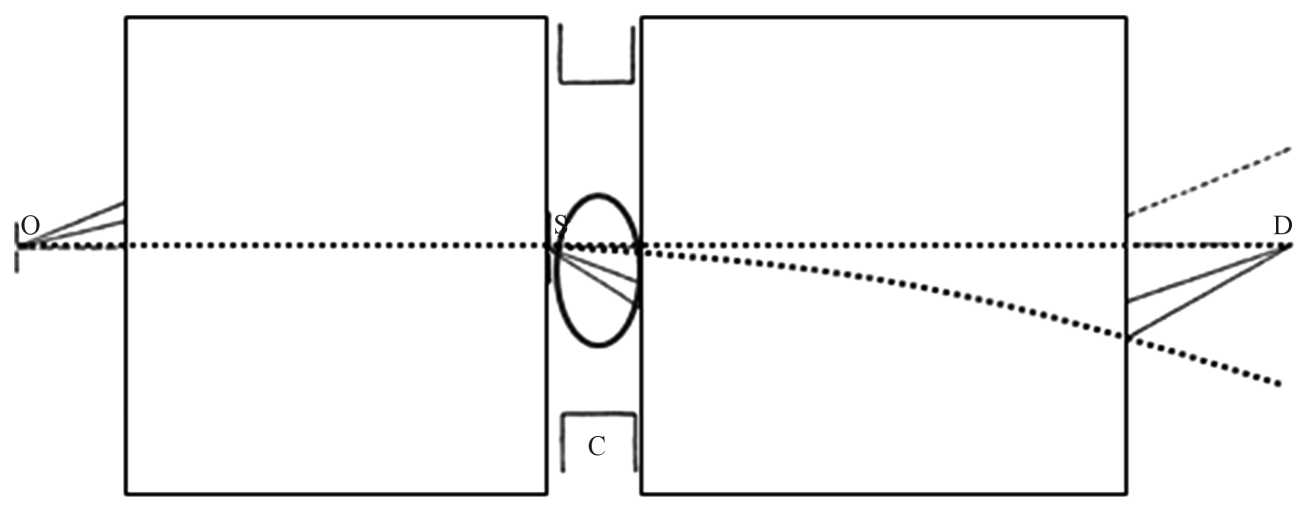

Figure 2. The dotted line path shows the trajectory change of the fraction of the molecular beam that is removed from the initial prepared state when passing thought the resonant cell. 
the fraction of the molecular beam that is removed from the initial prepared state does not reach the detector. But the important thing is: differently to the previous method, it happens without using non-homogeneous fields. Obviously, the trajectory change has to be explained without considering the force provided by the field gradient. There must be another molecular feature that explains the depletion. It looks as though the linear momentum conservation principle were not satisfied. These experiments suggest that a force depending on other fundamental magnitude of the particle, different from mass and charge must be taken into account.

\section{Looking for an Explanation}

In order to find out an explanation, let's consider the following case:

An electron is moving, with speed $\boldsymbol{v}$ constant in modulus, in a homogeneous magnetic field $\boldsymbol{B}$ where $\boldsymbol{v}$ is perpendicular to $\boldsymbol{B}$.

Its kinetic energy will be:

$$
E=\frac{1}{2} m v^{2}
$$

The electron, as is well known, describes a circular trajectory (in general case a helix) with a radius $r$, being:

$$
r=\frac{v}{\omega}
$$

and

$$
\omega=\frac{q \boldsymbol{B}}{m}
$$

due to the Lorentz force:

$$
\boldsymbol{F}=q \boldsymbol{v} \times \boldsymbol{B}
$$

On the other hand, as the electron has a magnetic moment, $\boldsymbol{\mu}$, and spin, $\boldsymbol{S}$, the presence of the magnetic field $\boldsymbol{B}$ produces a torque when interacting with the electron magnetic moment $\boldsymbol{\mu}$. The angle between $\boldsymbol{S}$ and $O_{z}$ (the direction of the magnetic field $\boldsymbol{B}$ ) remains constant but the spin $\boldsymbol{S}$ revolves about $O_{z}$ with angular velocity $\boldsymbol{\Omega}$. This phenomenon bears the name of Larmor precession.

The electron kinetic energy must increase with the energy due to spin precession. But it should be considered that the forces producing the torque are perpendicular to the precession motion and, as a consequence, do not modify the energy of the system. It looks like if the principle of energy conservation be violated.

\section{How to Solve This Dilemma?}

\subsection{First Option}

If the rotation around an axis is considered as origin of the spin, in a classic (and impossible) interpretation, one could imagine the electron rotating in a slowly way and offsetting the increase in energy due to the precession movement.

But, as it is well known, the spin is a quantized quantity; its modulus is constant and immutable. This option is, as a consequence, not acceptable.

\subsection{Second Option}

Let us consider now if, in our case, helicity is a constant of motion. Helicity, $\xi$, is defined as the scalar product of linear momentum and the spin:

$$
\xi=(m v) \cdot S
$$

where $\boldsymbol{S}$ is understood as the classic equivalent of the quantum spin and, as a consequence, $|\boldsymbol{S}|$ remains constant.Is this hypothesis consistent with Quantum Mechanics? Let us consider an electron in a uniform magnetic field $\boldsymbol{B}$, and let us choose the $O_{z}$ axis along $\boldsymbol{B}$. The classical potential energy due to electron magnetic moment $\boldsymbol{\mu}$ is then: 


$$
W=-\boldsymbol{\mu} \cdot \boldsymbol{B}=-\mu_{z} B=-\gamma B S_{z}
$$

where $B$ is the modulus of the magnetic field. Let us set:

$$
\omega_{0}=-\gamma B=\Omega
$$

$\Omega$ being the classical angular precession velocity. As is well known, $\omega_{0}$ has dimensions of the inverse of a time, that is, of an angular velocity. If we replace $S_{z}$ by the operator $S_{z}$ the classic energy becomes an operator; the Hamiltonian $H$ which describes the evolution of the spin of the electron in the field $\boldsymbol{B}$ is:

$$
H=\omega_{0} s_{z}
$$

Since this operator is time independent, solving the corresponding Schrödinger equation amounts to solving the eigenvalue equation of $H$. We immediately see that the eigenvectors of $H$ are those of $s_{z}$ (see [8] [9]):

$$
\begin{aligned}
& H|+\rangle=+\frac{\hbar \omega_{0}}{2}|+\rangle \\
& H|-\rangle=-\frac{\hbar \omega_{0}}{2}|-\rangle
\end{aligned}
$$

There are therefore two energy levels, $E_{+}=+\frac{\hbar \omega_{0}}{2}$ and $E_{-}=-\frac{\hbar \omega_{0}}{2}$. Their separation $\hbar \omega_{0}$ is proportional to the magnetic field and define a single "Bohr frequency"

$$
v_{+-}=\frac{1}{h}\left(E_{+}-E_{-}\right)=\frac{\omega_{0}}{2 \pi}
$$

Is it possible to distinguish, in a uniform magnetic field $\boldsymbol{B}$, which electrons are the state $|+\rangle$ and which are the state $|-\rangle$ ? The answer is no. Their behavior inside the field is exactly the same.

But, nevertheless, if we introduce an oscillating magnetic field $H_{1}$ with a frequency resonant with the transition $v_{+-}=\frac{1}{h}\left(E_{+}-E_{-}\right)=\frac{\omega_{0}}{2 \pi}$, then it will be possible to distinguish both states by the difference in their trajectories, see [10].

\section{Larmor Precession}

We will find, in Quantum Mechanics, the phenomenon equivalent to that described for a particle with classic magnetic moment and spin when moving in a uniform magnetic field $\boldsymbol{B}$ and which bears the name of Larmor precession.

Let us assume that, at time $t=0$, the spin is in the state

$$
|\chi(0)\rangle=\cos \frac{\theta}{2} \mathrm{e}^{-i \phi / 2}|+\rangle+\sin \frac{\theta}{2} \mathrm{e}^{i \phi / 2}|-\rangle
$$

To calculate the state $|\chi(t)\rangle$ in an arbitrary state $t>0$ and as $|\chi(0)\rangle$ is already expanded in terms of the eigenstates of the Hamiltonian we will obtain

$$
|\chi(t)\rangle=\cos \frac{\theta}{2} \mathrm{e}^{-\frac{i \phi}{2}} \mathrm{e}^{-i E_{+}+\frac{t}{\hbar}}|+\rangle+\sin \frac{\theta}{2} \mathrm{e}^{i i \phi / 2 e^{-i E}-\frac{t}{\hbar}}|-\rangle
$$

Or, using the values of $E_{+}$and $E_{-}$:

$$
|\chi(t)\rangle=\cos \frac{\theta}{2} \mathrm{e}^{-\frac{i\left(\phi+\omega_{0} t\right)}{2}}|+\rangle+\sin \frac{\theta}{2} \mathrm{e}^{+\frac{i\left(\phi+\omega_{0} t\right)}{2}}|-\rangle
$$

The presence of the magnetic field $\boldsymbol{B}$ therefore introduces a phase shift, proportional to time, between the coefficients of the kets $|+\rangle$ and $|-\rangle$.

Comparing the equation (14) for $|\chi(t)\rangle$ with that for the eingenket $|+\rangle_{u}$ for the observable $\boldsymbol{S} \cdot \boldsymbol{u}$

$$
|+\rangle_{u}=\cos \frac{\theta}{2} \mathrm{e}^{-i \phi / 2}|+\rangle+\sin \frac{\theta}{2} \mathrm{e}^{+\frac{i \phi}{2}}|-\rangle
$$


We see that the direction $u(t)$ along which the component is $+\frac{\hbar}{2}$, with certainty, is defined by the polar angles:

$$
\left\{\begin{array}{l}
\theta_{t}=\theta=\text { Cte } \\
\phi(t)=\phi+\omega_{0} t
\end{array}\right.
$$

that coincides with the direction along which the classic spin should be pointing out.

The angle between $u(t)$ and $O_{z}$ (the direction of the magnetic field $\boldsymbol{B}$ ) therefore remains constant, but $u(t)$ revolves around $O_{z}$ with angular velocity $\boldsymbol{\omega}_{0}=\boldsymbol{\Omega}$ proportional to the magnetic field. And the mean values of $S_{x}, S_{y}$ and $S_{z}$ behave like the components of a classical angular momentum of modulus constant $\frac{1}{2} \hbar$ undergoing Larmor precession.

\section{Helicity as a Constant of Motion}

As is well known, helicity is not, in general, a constant of motion. The reason is that helicity operator does not commute, in general, with the Hamiltonian H. Nevertheless, it will be proven that, at least for the interaction here considered (Larmor), the helicity eigenvalue is conserved along the electron's (classical) trajectory.

We redefine now the helicity, $\xi$, in order that its eigenvalues be \pm 1 , as $\xi=\sigma \hat{v}$, where $\hat{v}=\frac{v}{v}$ and $s=\frac{\hbar}{2} \boldsymbol{\sigma}$. The initial velocity of the electron is $v=v_{0}(\cos \phi, \sin \phi, 0)$, and we assume the initial spin state of the electron to be an eigenstate of the helicity with eigenvalue +1 , which is given in equation (12), with $\theta=\frac{\pi}{2}$ that is:

$$
|\chi(0)\rangle=\frac{\sqrt{2}}{2}\left(\mathrm{e}^{-\frac{i \phi}{2}}|+\rangle+\mathrm{e}^{\frac{i \phi}{2}}|-\rangle\right)
$$

At the time $t$ the velocity of the electron is, as it is known,

$$
v(t)=v_{0}\left[\cos \left(\phi+\omega_{0} t\right), \sin \left(\phi+\omega_{0} t\right), 0\right]
$$

where $\omega_{0}$ is given in Equation (7). According to Equations (15) and (16), with $\theta=\frac{\pi}{2}$, at time $t$ the spin state is:

$$
|\chi(t)\rangle=\frac{\sqrt{2}}{2}\left(\mathrm{e}^{-\frac{i\left(\phi+\omega_{0} t\right)}{2}}|+\rangle+\mathrm{e}^{+\frac{i\left(\phi+\omega_{0} t\right)}{2}}|-\rangle\right)
$$

and the helicity at time $t, \xi(t)=\boldsymbol{\sigma} \hat{v}(t)$,

$$
\xi(t)=\sigma_{x} \cos \left(\phi+\omega_{0} t\right)+\sigma_{y} \sin \left(\phi+\omega_{0} t\right)
$$

Now, taking into account that,

$$
\sigma_{x}(|+\rangle,|-\rangle)=(|-\rangle,|+\rangle) ; \sigma_{y}(|+\rangle,|-\rangle)=(i|-\rangle,-i|+\rangle) ;
$$

We easily obtain:

$$
\xi(t)|\chi(t)\rangle=|\chi(t)\rangle
$$

This shows that $|\chi(t)\rangle$ is an eigenstate of the helicity of eigenvalue +1 ; in other words, helicity is conserved along the electron's (classical) trajectory.

\section{Consequences}

It has been proven, for cases here considered, that helicity is a constant of motion. As a consequence of this result, the linear momentum $m v$ must have the same precession angular velocity (Larmor angular velocity) $\boldsymbol{\Omega}$ than the spin $\boldsymbol{S}$. The equation of motion describing the linear momentum evolution must be then equivalent of the 
equation of motion which describe the evolution of the spin $\boldsymbol{S}$. This means that:

$$
\begin{gathered}
\left(\frac{\mathrm{d} \boldsymbol{S}}{\mathrm{d} t}\right)_{\text {Inertial }}=\boldsymbol{S} \times \boldsymbol{\Omega} \\
\left(\frac{\mathrm{d} m \boldsymbol{v}}{\mathrm{d} t}\right)_{\text {Inertial }}=m \boldsymbol{v} \times \boldsymbol{\Omega}
\end{gathered}
$$

It is concluded the particle will be under a central acceleration, $\boldsymbol{a}=\boldsymbol{v} \times \boldsymbol{\Omega}$ perpendicular to $\boldsymbol{v}$. The particle is then under a central force:

$$
\boldsymbol{F}=m \boldsymbol{v} \times \boldsymbol{\Omega}
$$

This kind of forces related with the spin will be designed as Lorentz-like forces. In this case, the trajectory will be a circular one. The radius will be:

$$
R=\frac{|v|}{|\mathbf{\Omega}|}
$$

And its kinetic energy:

$$
E=\frac{1}{2} I_{z} \Omega^{2}=\frac{1}{2} m R^{2} \Omega^{2}=\frac{1}{2} m v^{2}
$$

which is equal to the initial one shown in Equation (2). The force (Equation (24)) is the responsible of the electron circular trajectory inside the field $\boldsymbol{B}$ and should be related to the spin $\boldsymbol{S}$ of the electron.

In conclusion, as helicity is, in those cases, a constant of motion, the particle is under a Lorentz-like force and the principles of conservation are not violated.

\section{Electron in a Magnetic Field}

If the case of an electron in a magnetic field is considered, then the force due to the spin of the electron will be:

$$
\boldsymbol{F}=m \boldsymbol{v} \times \mathbf{\Omega}
$$

where $\boldsymbol{\Omega}$ is the spin Larmor precession velocity around $O_{z}$. But is known that:

$$
\boldsymbol{\Omega}=\frac{|\boldsymbol{\mu}| \boldsymbol{B}}{|\boldsymbol{S}|}=\frac{g q \boldsymbol{B}}{2 m}
$$

Substituting in Equation (24) the expression for the force acting on the particle is obtained. This force has its origin on the spin. This expression is:

$$
\boldsymbol{F}=\frac{m g}{2 m} q \boldsymbol{v} \times \boldsymbol{B}
$$

As for an electron $g=2$, the final result is:

$$
\boldsymbol{F}=q \boldsymbol{v} \times \boldsymbol{B}
$$

Surprisingly this expression for the Lorentz-like force related to the spin coincides, formally, with that known as Lorentz force related to the charge. Considering the spin as responsible of the Lorentz-like force, a new deflection mechanism has been proposed (see [10]). The equations of motion for a system with intrinsic angular momentum when applying torques are described and, according with the theory, when the frequency of the oscillating field coincides with a transition resonant frequency (Larmor frequency), the molecules that change their state from the original one are removed from their trajectories and, as a consequence, do not reach the detector and the corresponding signal decreases.

\section{New Experimental Proposal}

In 1939 Alvarez and Bloch [11] measured the neutron magnetic moment by using a neutron beam passing through a resonant unit. Neutrons from the $\mathrm{Be}+\mathrm{D}$ reactions were slowed to thermal velocities and diffused down a Cadmium lined tube through the water tank to the polarizer magnet, $\mathrm{B}_{\mathrm{A}}$. After passing through the reso- 
nant unit that consists of two, a static and an oscillating, fields and the analyser magnet, $\mathrm{B}_{\mathrm{B}}$ they were detected in a $\mathrm{BF}_{3}$ chamber. The polarizer $\mathrm{B}_{\mathrm{A}}$ and analyser $\mathrm{B}_{\mathrm{B}}$ are strongly magnetized iron pieces. A neutron resonant dip is observed in the signal of the neutron beam when the oscillating resonant frequency corresponding to the transition between the two states up and down is reached. According to the previous theoretical description and recently results obtained for $\mathrm{NO}_{2}$, NO, NO dimer, $\mathrm{H}_{2}$ and $\mathrm{BaFCH}_{3}$ cluster, if the Álvarez and Bloch experiment is carried out without using analyser magnet $\mathrm{B}_{\mathrm{B}}$, we anticipate that the experimental results will be the same as those obtained by Álvarez and Bloch in the experiment of 1939.

According to the new explanation, the trajectory change takes place when neutrons pass through the resonant unit and the oscillating field is tuned to a transition resonant frequency between two states, up and down, of the spinof the neutron. In case of Álvarez and Bloch experiment, they used a magnetic field for the neutron resonance of 622 Gauss and a resonant frequency of oscillator of 1843 kilocycles.

\section{Summarizing}

- Some recent experimental results have reported the observation of molecular beam depletion when molecules of a pulse interact with a homogeneous static electric or magnetic field and an oscillating field (RF).

- In absence of non-homogeneous fields it is not possible to use the force provided by the field gradient interaction with the molecular dipole in order to explain this depletion.

- A unknown force depending on other fundamental magnitude of the particle, different of mass and charge must be considered.

\section{Conclusions}

To the best of our knowledge, it seems that existence of forces described in this paper, related with the spin of the particles, is the more adequate way to explain, from a theoretical point of view, the experimental result here considered. These forces are called Lorentz-like forces.

However, more experimental works are needed to support this conclusion. In this sense the experiment with neutrons, suggested in our proposal, is a very good example of a relevant experiment to be carried out.

\section{Acknowledgements}

The author is very grateful to prof. José L. Sánchez Gómez, Universidad Autónoma de Madrid and to Prof. Ramón Fernández Álvarez-Estrada, Universidad Complutense de Madrid, for useful discussions.

\section{References}

[1] Ramsey, N.F. (1990) Molecular Beams. Oxford University Press, Oxford.

[2] Rabi, I.I. (1935) Physical Review, 49, 324. http://dx.doi.org/10.1103/PhysRev.49.324

[3] Rabi, I.I., Millman, S., Kusch, P. and Zacharias, J.R. (1939) Physical Review, 55, 526. http://dx.doi.org/10.1103/PhysRev.55.526

[4] González Ureña, A., Gamo, L., Gasmi, K., Cáceres, J.O., De Castro, M., Skowronek, S., Dorado, M., Morales Furió, M., Pérez, J.L. and Sánchez Gómez, J.L. (2001) Chemical Physics Letters, 341, 495. http://dx.doi.org/10.1016/S0009-2614(01)00529-2

[5] Montero, C., González Ureña, A. and Morato, M. (2003) European Journal of Physics D, 26, 261-264. http://dx.doi.org/10.1140/epjd/e2003-00259-5

[6] Gasmi, K., Gonzálvez, A.G. and González Ureña, A. (2010) Journal of Physical Chemistry A, 114, 3229-3236. http://dx.doi.org/10.1021/jp909398w

[7] Montero, C., García Ureña, J. and Dorado, M. (2014) New Transition Mechanism: The Shaking Effect. http://arxiv.org/abs/1402.0203

[8] Dorado González, M. (1997) Anales de Física, 93, 105-120. arXiv:physics/0101085

[9] Cohen Tannoudji, C., Diu, B. and Laloë, F. (2005) Quantum Mechanics. Vols. I and II. Wiley-VCH, Hoboken.

[10] Dorado González, M. (2013) Dinámica de sistemas con espín: Un nuevo enfoque. Fundamentos y aplicaciones (in Spanish). ADI Servicios Editoriales.

[11] Alvarez, L.W. and Bloch, F. (1940) Physical Review, 57, 111. http://dx.doi.org/10.1103/PhysRev.57.111 
Scientific Research Publishing (SCIRP) is one of the largest Open Access journal publishers. It is currently publishing more than 200 open access, online, peer-reviewed journals covering a wide range of academic disciplines. SCIRP serves the worldwide academic communities and contributes to the progress and application of science with its publication.

Other selected journals from SCIRP are listed as below. Submit your manuscript to us via either submit@scirp.org or Online Submission Portal.
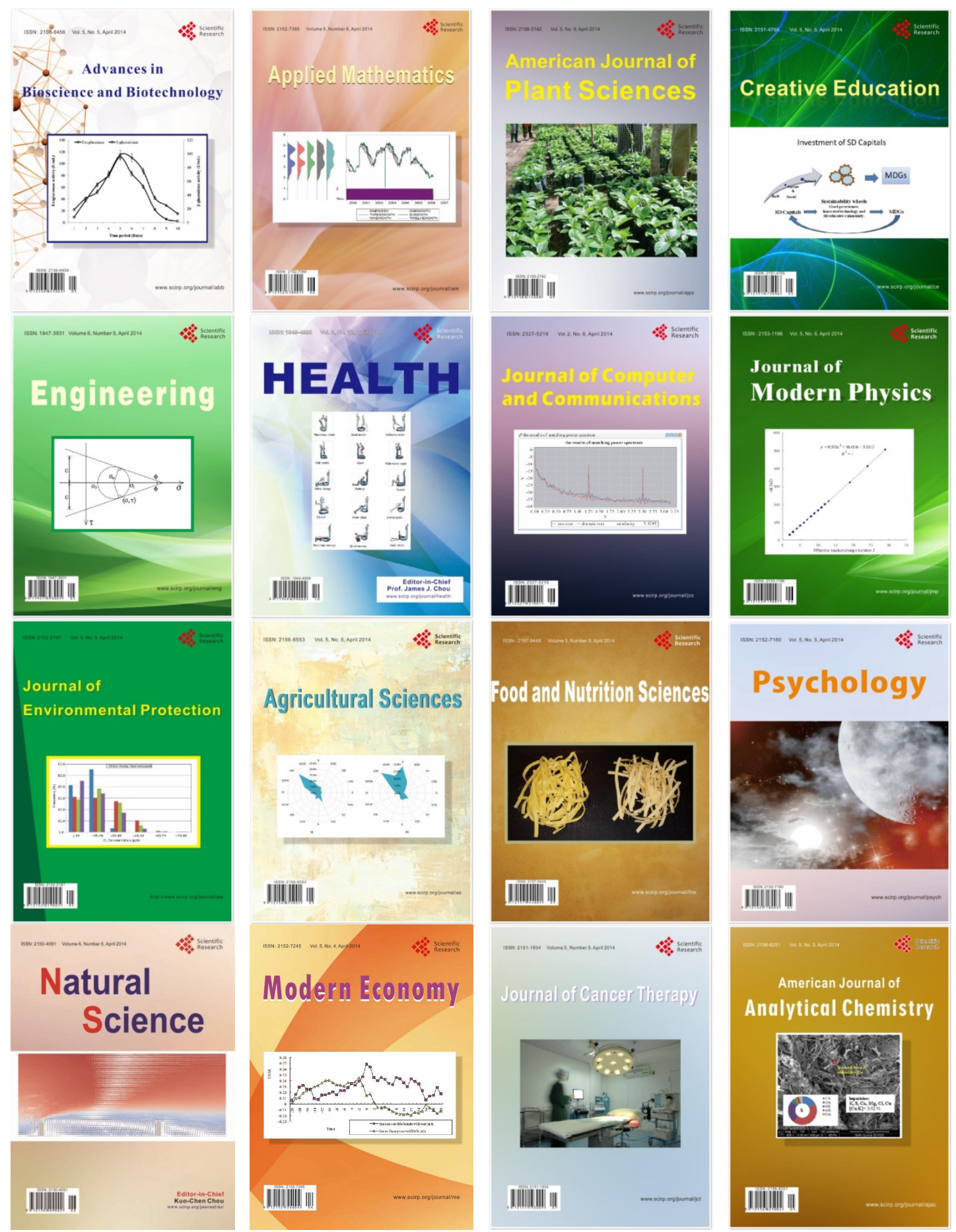